\title{
Response-cost punishment with pigeons: Further evidence of response suppression via token loss
}

\author{
Bethany R. Raiff, Christopher E. Bullock, and Timothy D. Hackenberg \\ University of Florida, Gainesville, Florida
}

\begin{abstract}
Four pigeons responded on a two-component multiple token-reinforcement schedule, in which tokens were produced according to a random-interval 30 -sec schedule and exchanged according to a variable-ratio 4 schedule in both components. To assess the effects of contingent token loss, tokens were removed after every second response (i.e., fixed-ratio 2 loss) in one of the components. Response rates were selectively lower in the loss components relative to baseline (no-loss) conditions, as well as to the within-condition no-loss components. Response rates were decreased to a greater degree in the presence of tokens than in their absence. To control for the effects of changes in the density of token and food reinforcement, two parts consisted of additional conditions where food density and token loss were yoked to those in a previous loss condition. In the yoked-food condition, tokens were produced as usual in both components, but the overall density of food reinforcement in one of the components was yoked to that obtained during a previous token-loss condition. In the yoked token-loss condition, tokens were removed during one component of the multiple schedule at a rate that approximately matched the obtained rate of loss from a previous token-loss condition. Response rates in these yoked components were less affected than those in comparable loss components, despite similar densities of token, exchange, and food reinforcement. On the whole, the results support the conclusion that contingent token loss serves as an effective punisher with pigeons.
\end{abstract}

Response-cost punishment procedures involve removal of reinforcers contingent on a response (Azrin \& Holz, 1966). Response-cost contingencies are pervasive in everyday life (e.g., monetary fines), and are frequently included in applied treatment packages involving token economies. Moreover, response-cost contingencies provide a way of examining punishment in terms that are conceptually analogous to reinforcement, as symmetrical changes along a common dimension via the addition or subtraction of tokens. Despite the practical and theoretical significance of response cost, the phenomenon is poorly understood.

Several studies have shown that response-cost contingencies, compared with no-cost conditions, suppress responding (Munson \& Crosbie, 1998; O’Donnell, Crosbie, Williams, \& Saunders, 2000; Weiner, 1962, 1963, 1964), supporting the notion that contingent reinforcement loss constitutes an effective form of punishment. Nearly all of the laboratory research on response cost, however, has been conducted with human subjects. This is likely due to the relative dearth of research with nonhumans on token reinforcement procedures, a precondition for arranging response-cost contingencies; that is, without some system in place for earning, accumulating, and exchanging tokens for other reinforcers, response-cost procedures are not possible. Although prior research with chimpanzees (Kelleher, 1958), rats (Malagodi, Webbe, \& Waddell, 1975), and pigeons (Foster, Hackenberg, \& Vaidya, 2001) has shown that behavior can be established and maintained under a variety of token-reinforcement procedures, token loss has not until recently been an explicit focus of research.

A practical impediment to such work is a suitable set of procedures by which tokens can be removed. Prior work in the token reinforcement realm with chimpanzees and rats has used manipulable tokens (e.g., poker chips, marbles) that are physically in a subject's possession until they are exchanged for food. Although such handling may enhance the reinforcing efficacy of the tokens, it poses special problems regarding token loss: Once in a subject's possession, a token (or tokens) cannot easily be removed. What is needed is a system by which a token (such as point displays used in experiments with human subjects) can be as easily presented as removed.

This type of nonmanipulable token reinforcement system has been developed over the past several years in laboratory research with pigeons (Bullock \& Hackenberg, 2006; Foster \& Hackenberg, 2004; Foster et al., 2001; Hackenberg \& Vaidya, 2003; Jackson \& Hackenberg, 1996). In this arrangement, the tokens consist of stimulus lamps arrayed in a horizontal row above the response keys in a standard conditioning chamber for pigeons. Each token is worth a predetermined amount of food (e.g., 2-sec food delivery) for which it can be exchanged during distinct exchange periods. Tokens are earned according to one schedule (the token-production schedule) and exchanged for food according to a second schedule (the exchange-production schedule). Behavior

B. R. Raiff, raiffbr@ufl.edu 
under these conditions closely resembles that seen under token-reinforcement schedules in other species with manipulable tokens (Kelleher, 1966). Unlike research with manipulable tokens, however, nonmanipulable tokens can be removed as easily as they can be presented, simply by extinguishing rather than illuminating a token lamp.

Using this type of token system, Pietras and Hackenberg (2005) arranged a response-cost punishment contingency in the context of a two-component multiple schedule with pigeons. In both components, tokens were produced according to a random-interval schedule and exchanged for food according to a variable ratio schedule. A conjoint fixed-ratio (FR) schedule of token loss was superimposed on the token reinforcement schedule in one component. Responding was suppressed in the loss component (approximately $30 \%-40 \%$ of unpunished rates) but was largely unaffected in the no-loss component.

In response-cost procedures, removal of reinforcers necessarily decreases the overall density of reinforcement, introducing an alternative explanation of response suppression: Changes in response rate may be attributed to response-contingent token loss or to decreases in the rate of food delivery. To control for this, Pietras and Hackenberg (2005) conducted a second experiment in which the density of food reinforcement was held constant across loss and no-loss components. This was accomplished with a withinsession yoking procedure in which the density of food reinforcement in one component (the no-loss component) was yoked to that from the immediately preceding component (the loss component). If token loss contributes to responserate decrements above and beyond that of decreased food reinforcement, responding should be more suppressed in the loss than in the no-loss components. Consistent with a punishment interpretation, response rates generally decreased more rapidly and remained consistently lower in the loss than in the no-loss components.

The effects reported by Pietras and Hackenberg (2005) were most discernable in transition from baseline to the loss and yoked-loss conditions. With extended exposure, rates began to converge, as overall response rates in both components decreased to low levels. This may have been due to a more general weakening of behavior brought on by the steadily decreasing reinforcement rates in both components. Because the density of food reinforcement was yoked to that in a component with a stringent tokenloss contingency, the overall reinforcement rates decreased sharply in the first sessions, leveling off at fewer than 0.5 food deliveries/min (compared with 2 deliveries/ min during baseline conditions). The direct suppressive effects of the punishment contingency may therefore have been masked by indirect effects of a leaner schedule of reinforcement - a product of the within-session yoking procedure. Moreover, because the tokens and exchange stimuli, as conditioned reinforcers, depend on reliable pairings with food, the significant reductions in food density may have also devalued the tokens and exchange stimuli as conditioned reinforcers (thereby decreasing the punishing effects of their loss).

In the present study, we sought to examine more systematically the role of positive reinforcement in response- cost punishment contingencies. We replicated key conditions from the Pietras and Hackenberg (2005) study, but with procedures in which reinforcement density was yoked across conditions, rather than across components within a session. Like the Pietras and Hackenberg study, ours employed a two-component multiple schedule. Unlike the yoking conditions in their study, however, one of the two components in the present study involved no loss of reinforcement. This helped to ensure a sufficiently high reinforcement density, thereby maintaining the relationship among the tokens, the exchange stimuli, and food. At the same time, this no-loss component provided an ongoing baseline against which to assess the effects of the response-cost contingencies.

Two yoking conditions were included: (1) yoked food, in which the number of food reinforcers was yoked to the number of food reinforcers earned in a prior response-cost condition (tokens were produced and accumulated, but exchanged for the same number of food reinforcers as had been earned under loss conditions); and (2) yoked token loss, in which the mean rates of token loss were yoked to the rates of token loss from a prior response-cost condition (tokens were removed as in response-cost conditions, but losses were not contingent on behavior). In both yoking conditions, the frequency of exchange periods was held approximately constant at one every $2 \mathrm{~min}$, the mean rate under no-loss conditions. This was done to equate rates of exchange-period stimuli-themselves potential sources of conditioned reinforcement - to permit a clearer assessment of the relative contributions of tokens and food to the reductions in responding under token-loss conditions, and the degree to which response-decreasing effects of the token-loss contingency were therefore due to punishment per se, apart from concomitant changes in token and food reinforcement.

\section{METHOD}

\section{Subjects}

Four male White Carneau pigeons, designated 730, 1447, 774, and 702 , maintained at approximately $80 \%$ of their free-feeding weights, served as subjects. The pigeons had served previously in an experiment on token reinforcement. Subjects were housed in individual home cages with continuous access to water and health grit. The pigeon colony was on a 16.5:7.5-h light:dark cycle.

\section{Apparatus}

A custom-built three-key operant chamber, enclosed in a soundattenuating cubicle, was used to run experimental sessions. The chamber had inside dimensions of $33.75 \mathrm{~cm}$ long $\times 55 \mathrm{~cm}$ wide $\times$ $41.25 \mathrm{~cm}$ high. An intelligence panel along one wall was equipped with three keys, an array of tokens, a food receptacle, and a houselight. The three key lights $(2.5 \mathrm{~cm}$ in diameter) were equidistant from each other $(6 \mathrm{~cm}$ from center), with the left and right key lights positioned $8.5 \mathrm{~cm}$ from the side walls. The keys could be illuminated red, yellow, or green from the rear with 28 -W lights, and required approximately $0.17 \mathrm{~N}$ force to activate. Effective responses were accompanied by an audible click produced by a relay. Tokens were composed of an array of 12 red-capped lights $(28 \mathrm{~W} ; 1.5 \mathrm{~cm}$ in diameter) and were positioned $2 \mathrm{~cm}$ above the key lights, $1 \mathrm{~cm}$ apart; the leftmost and rightmost tokens were located $8.5 \mathrm{~cm}$ from their respective sidewalls. The food receptacle $(6 \mathrm{~cm}$ long $\times 6 \mathrm{~cm}$ wide $\times 5 \mathrm{~cm}$ high $)$ was located $22.5 \mathrm{~cm}$ below the center key and was illuminated by a yellow 
light whenever the food hopper was activated. A houselight $(28 \mathrm{~W})$ was located above the token array, centered $32.5 \mathrm{~cm}$ from the metal grate floor. A food hopper, filled with mixed grain, and a sonalert $(2,900 \mathrm{~Hz}, 65 \mathrm{~dB})$ were located outside the chamber but within the sound-attenuating cubicle. Speakers located in the room played white noise to mask extraneous sounds. A computer in an adjacent room, equipped with Med-PC software (MED Associates, East Fairfield, VT), was used to program experimental events and to collect data.

\section{Procedure}

Because pigeons had experience with token-reinforcement procedures, no pretraining was necessary. Sessions occurred at approximately the same time each day, 7 days per week, and began with a 5-min blackout period, after which the houselight and the left (token-production) key were illuminated. A two-component multiple schedule was used, whereby two independent schedules (hereafter, components) were arranged successively, each correlated with a distinct stimulus. In both components of the multiple schedule, a second-order token production schedule was in effect, during which pecks on the left (token-production) key produced tokens (from left to right on the token array) according to a random-interval (RI) 30 -sec schedule. Exchange periods, during which tokens were exchanged for food, were produced according to a variable-ratio-4 (VR4) schedule of token production; that is, when an average of 4 tokens (rectangular distribution ranging from 1 to 7) had been produced, an exchange period began. During exchange periods, the token-production key light was turned off and the center, exchange, key light was illuminated red. Each response on the exchange key removed one token (from right to left), turned off the houselight and center key light, and provided 2.5 -sec access to mixed grain. Exchange periods lasted $30 \mathrm{sec}$, which provided ample time to exchange all tokens earned during that cycle.

Each component was correlated with a green or yellow left key light and was presented twice each session (the first and third components were randomly selected). Components ended following the first exchange period after 6 min of token-production time. A 30-sec blackout occurred between components, during which all lights were off and responses had no programmed consequences. The experiment was divided into two parts, each consisting of a sequence of conditions constituting an $\mathrm{A}-\mathrm{B}-\mathrm{A}-\mathrm{C}$ experimental design. In both parts, the A (baseline) conditions involved no loss. The B (token loss) conditions involved a conjoint token-loss schedule superimposed on the token-production schedule in one of the two components. The $\mathrm{C}$ (yoking) conditions involved either yoked food density (Part 1) or yoked token loss (Part 2) in one of the two components. Table 1 shows the order of conditions and the number of sessions per condition for each pigeon. All conditions lasted a minimum of 15 sessions, until response rates during both components were deemed stable according to visual inspection. Response rates were considered stable when there were no discernible trends (i.e., no monotonic increases or decreases) across 3 successive sessions.

\section{Part 1}

Baseline (A). In the baseline condition, identical VR 4 (RI 30) token schedules operated in both components.

Token loss (B). The token loss condition involved arranging an FR 2 schedule of token loss conjointly with the token-production schedule in one component of the multiple schedule (hereafter the "loss" component). The VR 4 (RI 30) token schedule continued to operate in the other component (the "no-loss" component). Every second response in the loss component extinguished the rightmost token and produced a brief flash of the houselight and a tone. The FR 2 token-loss contingency restricted the number of loss-component tokens to one in this condition: The first response produced the token, and the second response subsequently removed it. Thus, it was only possible to produce exchange stimuli when the smallest element (1) in the VR 4 exchange-schedule distribution had been selected. Because this would reduce the rate of exchange stimuli, a randomtime (RT) 120-sec exchange schedule was superimposed on the VR 4 exchange schedule in the loss component. This held approximately constant the rate of exchange periods across components. If an exchange period was instituted and no tokens had been earned, the exchange period ended after $30 \mathrm{sec}$. The first row of Figure 1 depicts a hypothetical sequence of events during the loss component in the token-loss condition.

Yoked food (C). As with the token-loss condition, the VR 4 (RI 30) token schedule continued to operate in the no-loss component. In the yoked-food conditions (middle row of Figure 1), the probability of food resulting from a token exchange in the loss component was yoked to the average number of food presentations from the last 5 sessions of the most recent token-loss condition for that same pigeon. Note that in this condition, the loss component does not refer to actual tokens being lost, but rather to a yoked decrease in the density of food obtained during the same key-color components used during the loss component of the token-loss condition. For example, Pigeon 1447 exchanged an average of 4.6 tokens during an average of 9.2 exchange periods in the last 5 sessions of the loss component during the token-loss condition. The probability of exchanging a token for food during the loss component in the yokedfood condition was thus set to 0.5 (i.e., 4.6 tokens/ 9.2 exchanges $=$ $0.5)$. Therefore, when an exchange period was entered during loss components in this condition, the overall probability of exchanging a token for food was divided by the number of tokens available during that exchange period. For instance, if the overall probability of earning food for exchanging a token was set to 0.5 , and 5 tokens were available during the exchange period, each token was assigned an equal probability $(0.1)$ of food delivery (i.e., 0.5 probability of food $/ 5$ tokens $=0.1$ probability of food for each token). Table 1 shows the scheduled probabilities of an exchange response produc-

Table 1

Condition Sequence, Number of Sessions per Condition, and Yoked Values

\begin{tabular}{|c|c|c|c|}
\hline Pigeon & $\begin{array}{l}\text { Condition } \\
\text { Sequence }\end{array}$ & $\begin{array}{l}\text { Number of } \\
\text { Sessions }\end{array}$ & Yoked Value \\
\hline \multicolumn{4}{|c|}{ Part 1} \\
\hline \multirow[t]{4}{*}{1447} & Baseline & 22 & - \\
\hline & Token loss & 44 & - \\
\hline & Baseline & 32 & - \\
\hline & Yoked food & 40 & Probability $=.49$ \\
\hline \multirow[t]{4}{*}{730} & Baseline & 16 & - \\
\hline & Token loss & 20 & - \\
\hline & Baseline & 45 & - \\
\hline & Yoked food & 55 & Probability $=.23$ \\
\hline \multirow[t]{4}{*}{774} & Baseline & 17 & - \\
\hline & Token loss & 23 & - \\
\hline & Baseline & 21 & - \\
\hline & Yoked food & 32 & Probability $=.19$ \\
\hline \multirow[t]{4}{*}{702} & Baseline & 27 & - \\
\hline & Token loss & 30 & - \\
\hline & Baseline & 38 & - \\
\hline & Yoked food & 17 & Probability $=.32$ \\
\hline \multicolumn{4}{|c|}{ Part 2} \\
\hline \multirow[t]{4}{*}{1447} & Baseline & 40 & - \\
\hline & Token loss & 31 & - \\
\hline & Baseline & 61 & - \\
\hline & Yoked token loss & 33 & RT $48 \mathrm{sec}$ \\
\hline \multirow[t]{4}{*}{730} & Baseline & 34 & - \\
\hline & Token loss & 16 & - \\
\hline & Baseline & 26 & - \\
\hline & Yoked token loss & 60 & RT $60 \mathrm{sec}$ \\
\hline \multirow[t]{4}{*}{774} & Baseline & 34 & - \\
\hline & Token loss & 18 & - \\
\hline & Baseline & 15 & - \\
\hline & Yoked token loss & 18 & RT $40 \mathrm{sec}$ \\
\hline
\end{tabular}

Note-RT, random time. 


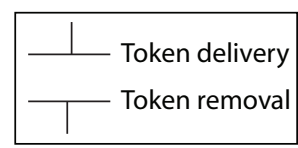

Exchange Period: $\quad$ Exchange Period:

"0 tokens present

"0 tokens exchanged
*1 token present

*1 token exchanged

Token Loss
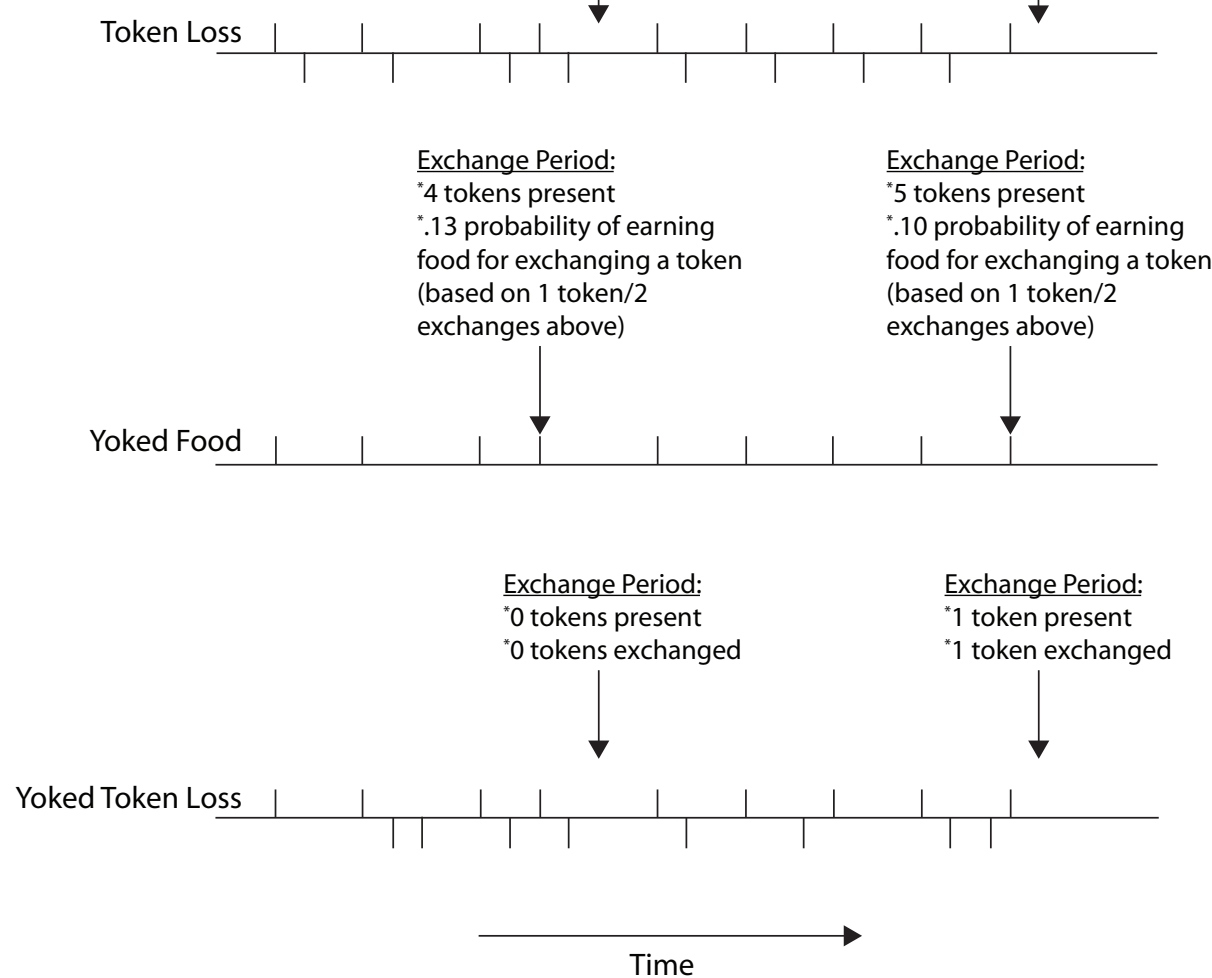

Figure 1. Schemata depicting hypothetical temporal distribution of token deliveries, token removals, and exchange periods, in the loss component of the token-loss, yoked-food, and yoked token-loss conditions. Token deliveries are denoted by vertical upticks, token removals by vertical downticks, and exchange periods by arrows. The yoked-food and the yoked token-loss scenarios are linked to the token-loss events shown in the first row.

ing food during an exchange period for each pigeon. Because the token-loss contingency was suspended in this yoked-food condition, the RT exchange schedule was deemed unnecessary. The sole exception was for Pigeon 774, for whom the RT schedule unintentionally remained in place, but this had no discernible effect on the obtained density of reinforcement (approximately $28 \%$ of exchanges during this component were initiated via the RT schedule).

\section{Part 2}

Baseline (A). Identical to baseline conditions described in Part 1. Token loss (B). Identical to token-loss conditions described in Part 1.

Yoked token loss $(\mathrm{C})$. In yoked token-loss conditions (see bottom row of Figure 1), the average rate of token loss from the last 5 sessions of the most recent token-loss condition was calculated and programmed as an RT (i.e., response-independent) token-loss schedule. The rate of loss was calculated by subtracting the total number of tokens exchanged from the total number of tokens earned, divided by the total amount of time spent in the loss component (Table 1 shows programmed RT schedules for each subject). If a token was scheduled for removal, but no token was available, the next earned token was removed immediately after presentation. Because this schedule would reduce the rate of access to the exchange period in a similar fashion as token-loss conditions, the RT 120-sec exchange schedule was in place.

For Pigeon 702, we were unable to replicate in Part 2 the original suppressive effects of the token-loss condition from Part 1, and therefore undertook a series of conditions to reestablish experimental control. Although we were able to eventually replicate the initial token-loss results, because this series of conditions did not include the critical yoked token-loss condition, data from these conditions are not presented here.

\section{RESULTS}

In the present study, punishment effects can be shown in at least two different ways: (1) as decreases in response rates relative to prepunishment conditions (i.e., acrosscondition comparisons), and (2) as decreases in response rates relative to other, no-punishment, components (i.e., within-condition comparisons). To facilitate comparisons across subjects and conditions, response-rate data were converted to a proportion of baseline.

To illustrate the across-condition comparisons, Figure 2 shows mean response rates for each pigeon across the final 5 sessions of the token-loss and yoked conditions, expressed as a proportion of the final 5 sessions from the immediately prior baseline condition. Values falling above and below the dotted line indicate, respectively, increases and decreases relative to pre-loss baseline 


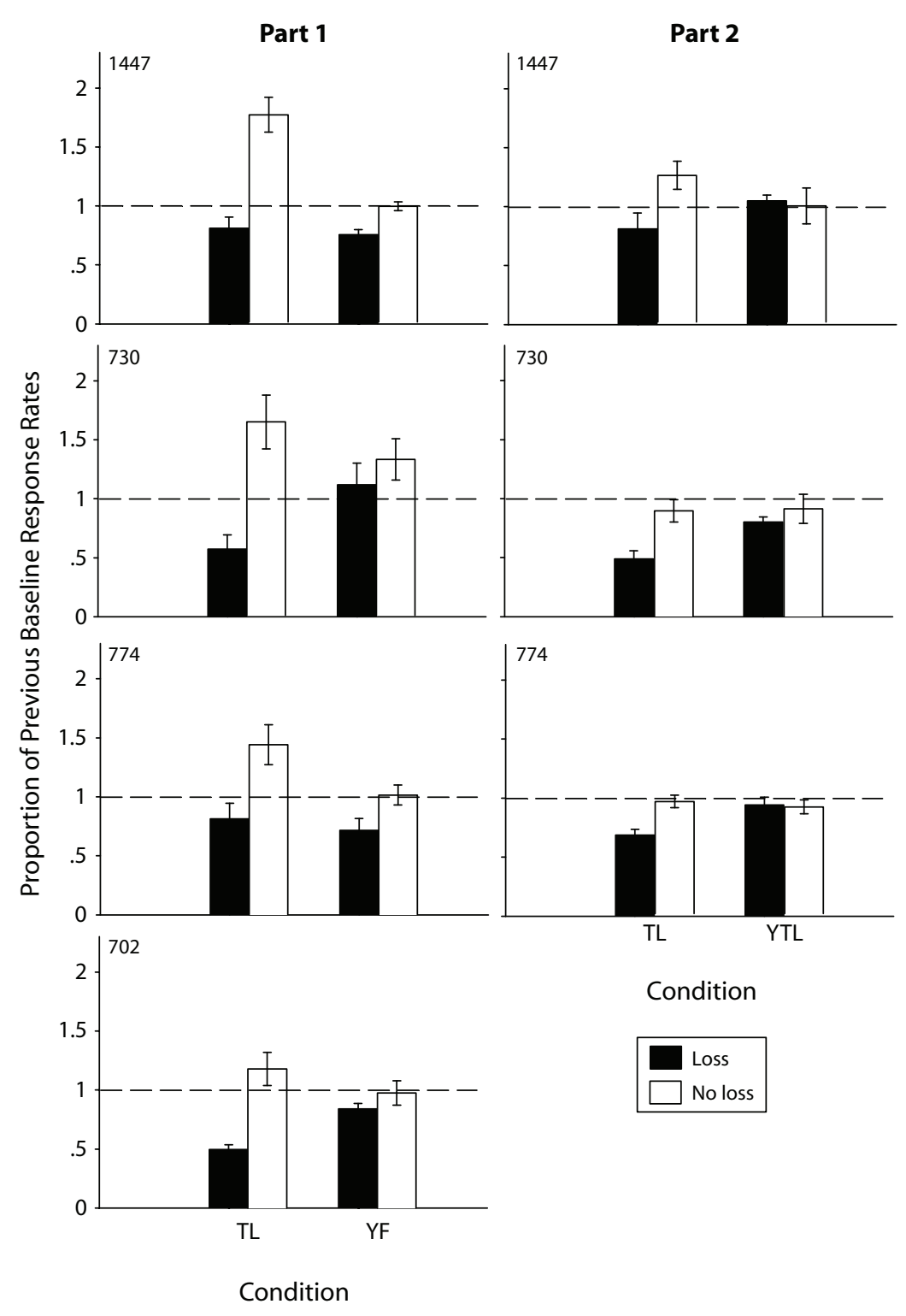

Figure 2. Across-condition comparisons. Mean response rates (and standard deviations) as a proportion of the immediately preceding baseline condition in the loss component (black) and the no-loss component (white). The left panels show proportions during Part 1 and the right panels proportions during Part 2. The labels TL, YF, and YTL denote token loss, yoked food, and yoked token loss, respectively.

responding. Considering first the data from Part 1 (left panels of Figure 2): Response rates in the initial tokenloss conditions decreased substantially in the loss component for all pigeons (mean change, $-33 \%$; range, -51 to -19). Although not shown here, the decreases in response rates in the loss component began early in the condition, in most cases evident in the initial 5 sessions. Interestingly, response rates increased in no-loss components (mean change, $+32 \%$; range, +15 to +43 ) during this condition, despite no change in the contingencies in that component. In the yoked-food condition, response rates decreased relative to baseline for 3 of 4 pigeons (1447, 774 , and 702), but to a lesser extent on average than under the token-loss conditions (mean change, $-16 \%$; range, -29 to +11 ), with only 2 of the 4 pigeons showing decreases comparable to those seen during the token-loss condition. When decreases were evident, they occurred more gradually than they did under token-loss conditions. The increases in no-loss component response rates observed in the token-loss conditions were less evident in these yoked-food conditions (mean change, $+7 \%$ ), with only one pigeon (730) showing such increases.

In Part 2 (right panels of Figure 2), response rates in the loss component of token-loss conditions again decreased relative to baseline (average change, $-34 \%$; range, -51 to -19). Unlike the initial exposure to token-loss conditions, however, no-loss component response rates were approximately equal to baseline on average, with increases evi- 
dent for only one pigeon (1447). During yoked token-loss conditions, response rates in both components approximated baseline levels (mean change, $-7 \%$; range, -17 to +5 ). In summary, token-loss conditions consistently decreased response rates relative to those in a previous baseline condition, yoked-food conditions decreased response rates relative to those in a baseline condition for some pigeons, but not others, and yoked token-loss conditions did not decrease response rates relative to those in a previous baseline condition at all.
Next, to investigate the presence of within-condition decreases in response rates as a function of contingent token loss, Figure 3 expresses loss component response rates relative to no-loss component response rates from the same condition for each pigeon according to the sequence in which the conditions were experienced. For Figure 3, values falling above the dotted line indicate increases in response rates in loss components relative to no-loss components, and values falling below the dotted line indicate decreases in response rates in loss compo-

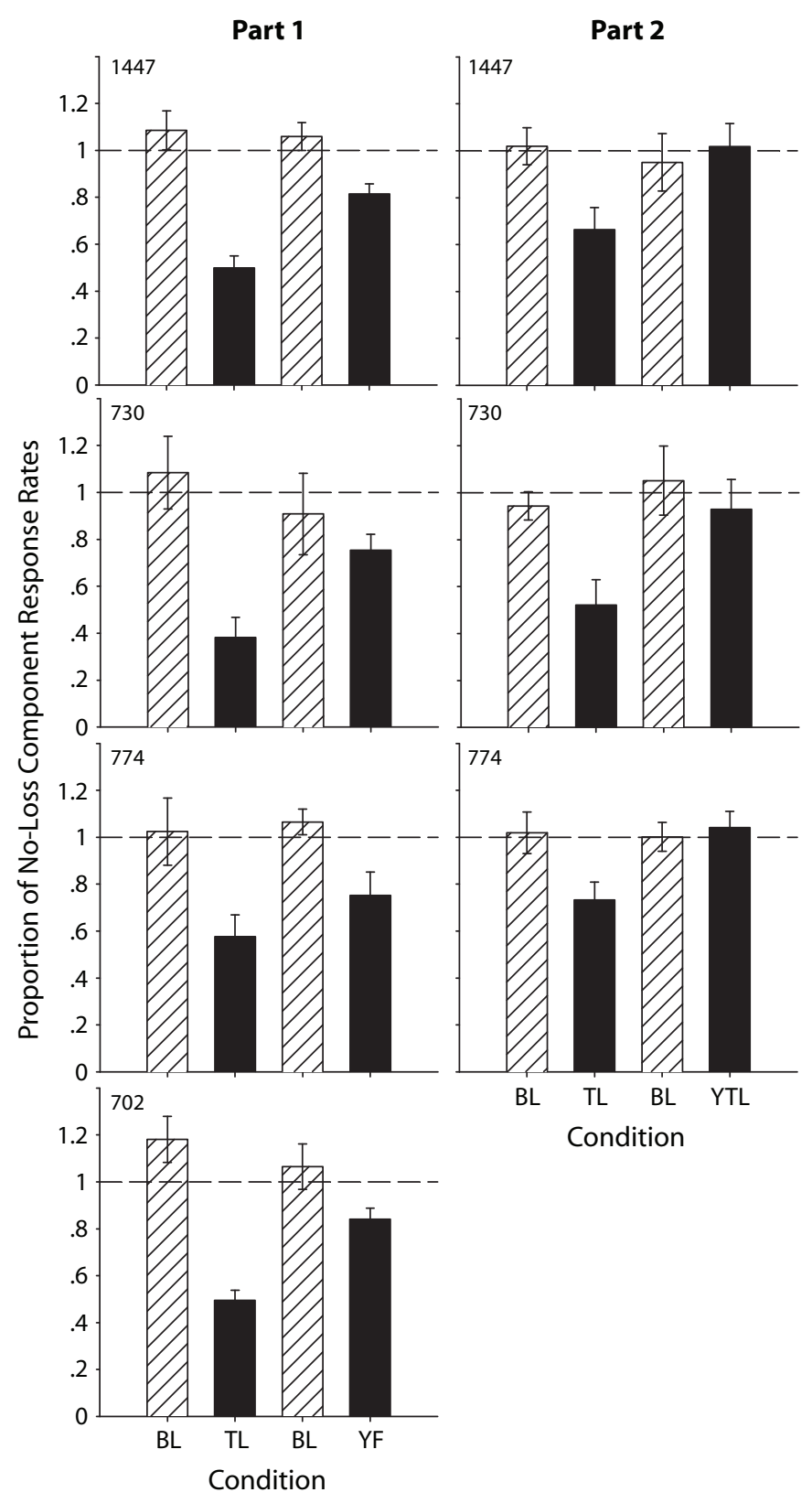

Figure 3. Within-condition comparisons. Mean response rates (and standard deviations) as a within-session proportion of the no-loss component for baseline (striped), token-loss, and yoked-control conditions (black). The left panel shows proportions during Part 1 and the right panel shows proportions during Part 2. The labels TL, YF, and YTL denote token loss, yoked food, and yoked token loss, respectively. 
nents relative to no-loss components. In baseline conditions (when no tokens were lost), the ratios of response rates in loss to no-loss components were close to 1.0 (mean, 1.03 across pigeons), indicating approximately equal responding in the two components. In Part 1 (left panels), the ratio was lower under token-loss conditions (mean, .49; range, .37-.57) than under yoked-food conditions (mean, .79; range, .75-.84), indicating greater within-condition response suppression under token-loss than under yoked conditions. Similarly, in Part 2, the ratio was lower under token-loss conditions (mean, .64; range, .52-.73) than under yoked token-loss conditions (mean, 1.00; range, .96-1.04). In summary, the token-loss procedure produced large and consistent within-condition decreases; the yoked-food conditions produced consistent but smaller within-condition decreases. The yoked tokenloss conditions did not produce systematic effects.

In response-cost procedures, punishment contingencies can only be enforced when there are tokens available for removal. It is therefore informative to examine differences in the local rates of responding when tokens are present (i.e., available for removal) and when tokens are absent. These comparisons can be conceptualized as withincomponent comparisons. Figure 4 shows responses per minute in the presence and absence of tokens during the token-loss and yoked token-loss conditions of Part 2. Considering first the data from token-loss conditions (left panels), response rates were substantially lower when tokens were present (mean, 9.5 responses/min; range, 8.5-10.5) than when they were absent (mean, 66.5 responses $/ \mathrm{min}$; range, 43.2-105.5). Such differences in local rates should only be expected, however, when tokens are removed contingent on a response, not when tokens are removed independently of a response. It is therefore instructive to compare the selective suppression of responding seen under token-loss conditions with the nondifferential responding seen in yoked token-loss conditions (right panels): 78.5 responses/min (range, 62.7-90.1) with tokens

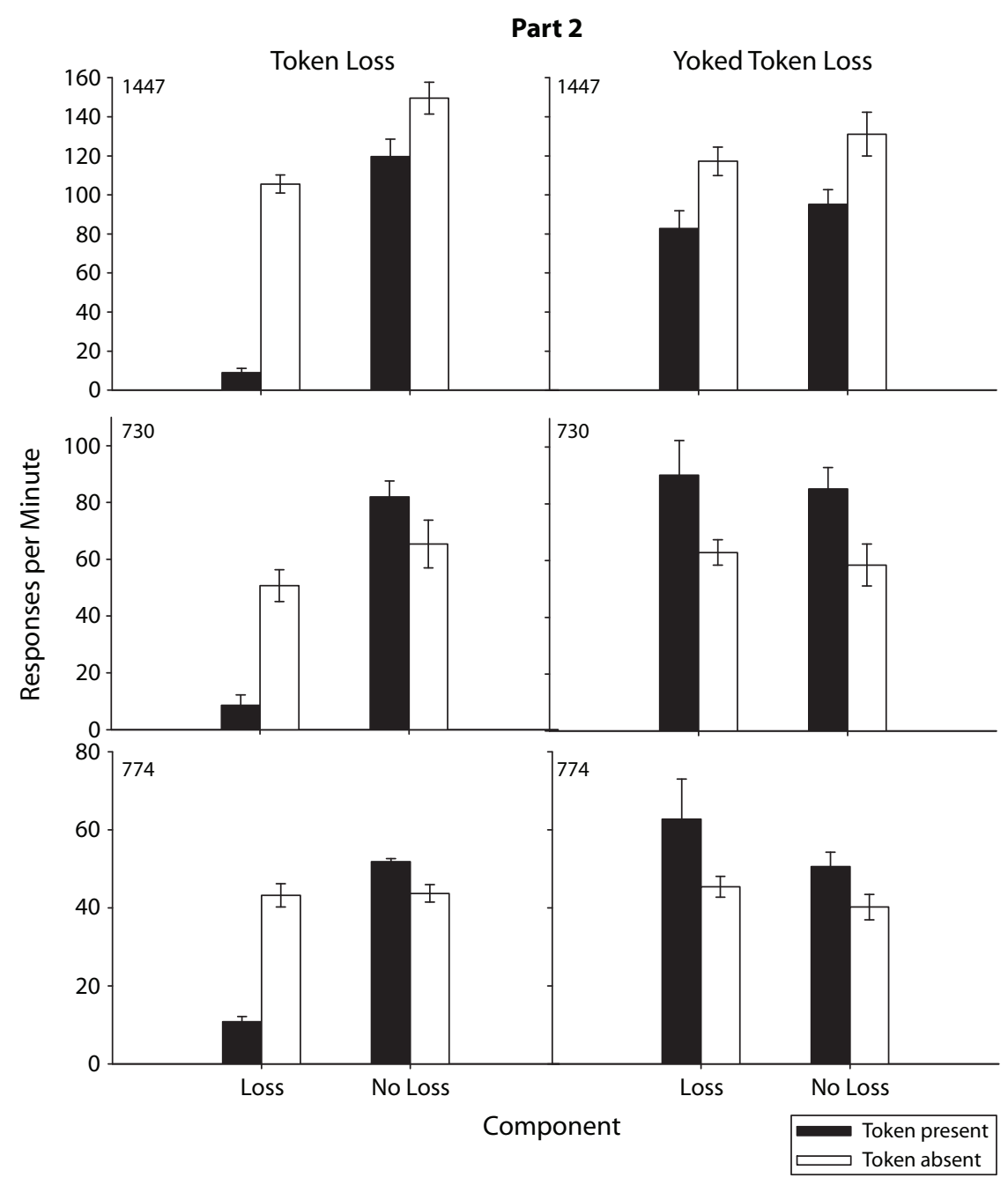

Figure 4. Within-component comparisons. Local response rates (and standard deviations) from the last 5 sessions of the token-loss (left panel) and yoked token-loss (right panel) conditions of Part 2. Shown are mean response rates from the loss and no-loss components in the presence (black) and absence (white) of tokens. 
present versus 75.1 responses/min (range, 45.4-117.2) with tokens absent. Although not shown here, the same pattern of results was observed in Part 1, with differential suppression in the presence of tokens during token-loss conditions only. Therefore, decreases in response rates during token-loss conditions can be accounted for almost entirely by suppression occurring when tokens were available for removal.

Historically, studies involving electric shock punishment have included an analysis of changes in response patterning via cumulative response records (e.g., Azrin, 1960a, 1960b). To better compare the present findings to those using electric shock, Figure 5 shows cumulative response records from the final session of the two token-loss condi- tions and the two yoked conditions. The data were selected as representative, with records from the pigeon showing the greatest (Pigeon 730) and least (Pigeon 774) suppression during token loss. In both token-loss conditions, the slopes were shallower in the loss component than in the no-loss component for both pigeons. Conversely, during the yokedfood (Pigeon 730) and yoked token-loss (both Pigeons 730 and 774) conditions, the slopes were approximately equal in the loss and no-loss components. The slopes of the records for Pigeon 774, on the other hand, were somewhat shallower during the loss components of the yoked-food condition, but to a lesser extent overall than during the token-loss condition; thus, the pattern of responding was less affected by punishment than was the slope.

\section{Part 1}

730 (Scale Ends at 900 Responses)
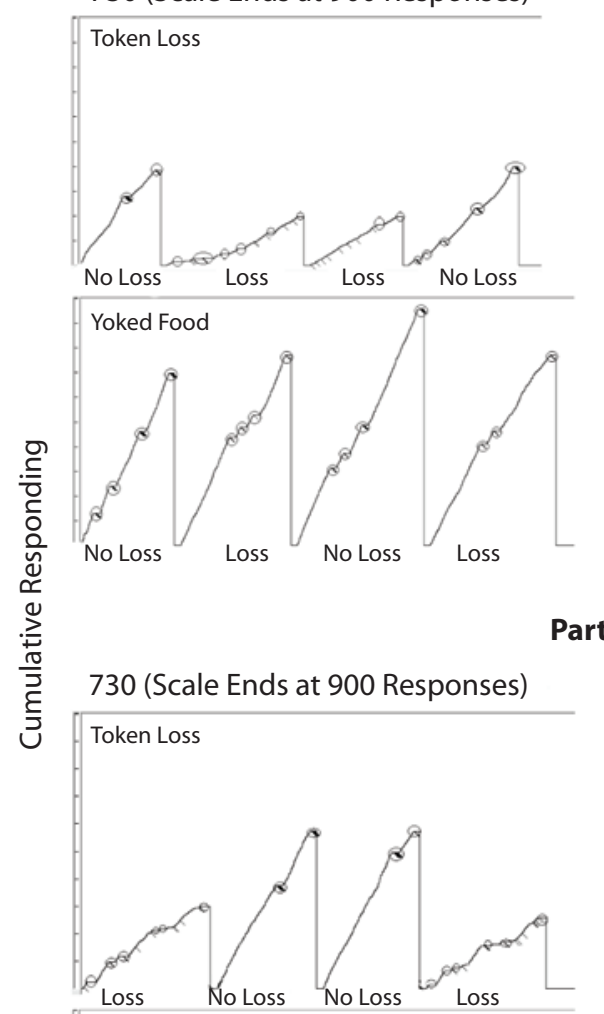

Yoked Token Loss
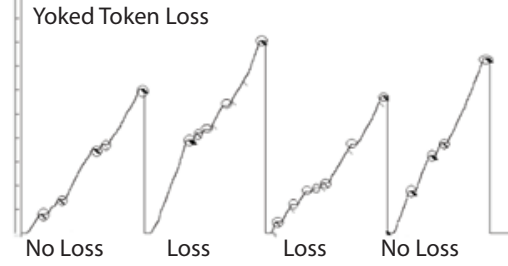

Components

Part 2
774 (Scale Ends at 650 Responses)
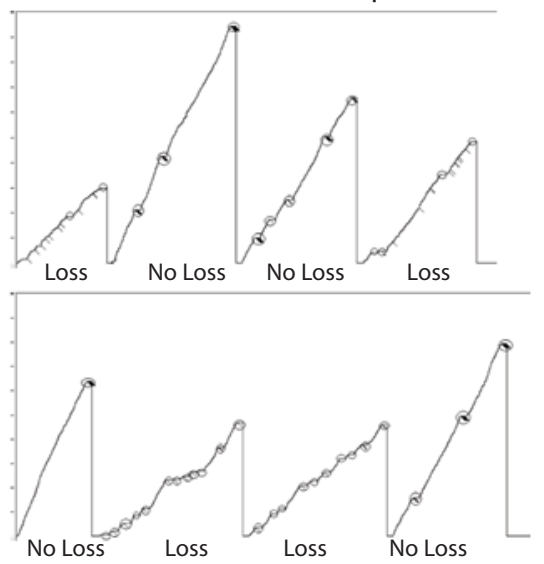

774 (Scale Ends at 650 Responses)

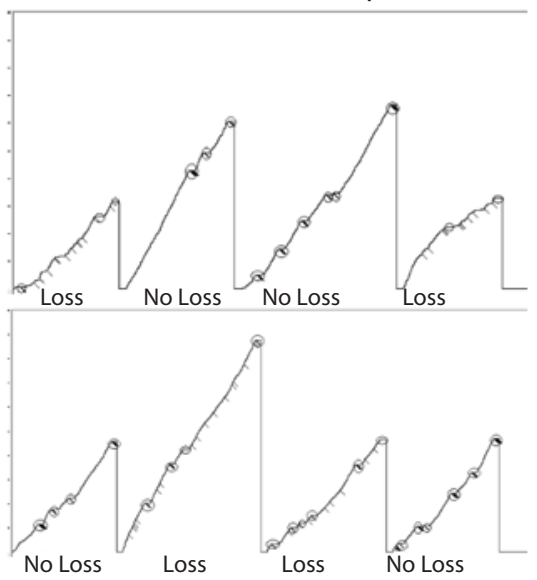

Figure 5. Within-session response patterns. Cumulative response records from the last session of the token-loss and yoked-food conditions of Part 1 (first and second rows), and the token-loss and yoked token-loss conditions of Part 2 (third and fourth rows) for Pigeons 730 and 774 (columns). Deflections in the record indicate when a token was removed (either response-dependent during token loss or response-independent during yoked token loss). Circled portions of the record indicate exchange periods, and here deflections of the pen represent responses during token exchange. Note that the scale ends with 900 responses for Pigeon 730 and the scale ends with 650 responses for Pigeon 774. 
Table 2 shows the mean response, reinforcement, token loss, and exchange rates in both components for each pigeon across the final 5 sessions of each condition. Approximately one exchange occurred every $2 \mathrm{~min}$ in both components of the baseline conditions and in the no-loss components of the token-loss and yoked conditions. Likewise, the rate of exchanges was approximately one every $2 \mathrm{~min}$ in the loss component of the yoked-food condition for all but Pigeon 774, who had a slightly higher rate of exchange (1.3 exchanges/min), due to the unintentional inclusion of the RT 120 -sec exchange schedule. The rate of exchanges during the loss components of the token-loss conditions was similar to the rate of exchanges during the loss components of the yoked token-loss conditions.

Under no-loss, reinforcement rate approximated the programmed rate of $2 / \mathrm{min}$ (Table 2). In Part 1 , in which rates of food reinforcement were matched across loss and yoked conditions, the reinforcement rates in loss components were lower under token-loss and yoked conditions than under noloss conditions (approximately $0.2 / \mathrm{min}$, on average), but not appreciably different from each other. In Part 2 , in which rates of token loss were matched across loss and yoked conditions, the rates of tokens lost during the loss component under yoked conditions were not discernibly different than under token-loss conditions (approximately 1.3/min, on average). In short, both yoking procedures worked as planned, with the overall density of food reinforcement (Part 1) and rate of token loss (Part 2) held approximately equal to that of the previous token-loss condition.

\section{DISCUSSION}

Contingent token loss produced substantial decreases in response rates relative to no-loss conditions in all 4 pigeons. The suppression of responding under token loss was assessed in three ways: (1) across conditions, by comparing response rates under token loss to rates under analogous conditions in the same component of the immediately prior baseline condition (Figure 2), (2) within conditions, by comparing response rates under token loss to rates in the no-loss component in the same condition (Figure 3), and (3) within components, by comparing local response rates in the presence versus the absence of tokens (Figure 4). Response rates were reduced by an average of $33 \%$ and $45 \%$ of no-loss rates by the across-condition and within-condition measures, respectively. Moreover, local response rates were differentially affected by the tokenloss contingencies, with local rates in the presence of tokens reduced by an average of $86 \%$ of local rates in the absence of tokens. Thus, when responding was suppressed in token-loss conditions, most of the suppression occurred in the presence rather than in the absence of tokens. Such differential suppression is consistent with a punishment account, insofar as the presence of tokens comprises a set of stimulus conditions correlated with the token-loss contingency, which only operates with tokens present. Thus, by all three measures, responding was consistently suppressed by token loss, supporting the conclusion that contingent token loss serves as an effective punisher with pigeons under laboratory conditions.
The results are consistent with those reported by Pietras and Hackenberg (2005), who also found response suppression by contingent token loss under conditions similar to those used here. Because token loss, by definition, reduces the density of food reinforcement, it is important in studies of this type to include control conditions to isolate the effects of token loss from concomitant changes in the density of food reinforcement. Pietras and Hackenberg accomplished this with a within-session yoking procedure, in which the density of food reinforcement in the no-loss component was yoked to that in the loss component in the same session. In general, the suppression was greater under loss than under no-loss conditions, but because the overall rate of food reinforcement was so low across both components, the effects may have been masked by a general weakening of behavior.

In the present study, we yoked the density of food reinforcement in yoked-food conditions to that from a preceding token-loss condition, while maintaining in the no-loss component an ongoing schedule of food reinforcement that preserved the relations between tokens, exchange stimuli, and food. In the yoked-food conditions (Part 1), response rates were reduced relative to baseline conditions, but less so than under contingent token loss. Because the food reinforcement rates were comparable in the two conditions, the difference between rates under token loss and yoked controls may be attributed to the rate-suppressing effects of the token-loss contingency apart from the reductions in the rate of food reinforcement.

Aside from reductions in food reinforcement, the mere loss of tokens may also have contributed to response suppression under token-loss conditions. We therefore included a condition in Part 2, in which rates of token loss were yoked to a prior token-loss condition. Response rates in these yoked token-loss conditions were affected little, if at all, by noncontingent token loss. Because the rates of token loss were similar to those in token-loss conditions but were not contingent on behavior, the effects of contingent loss were separable from the effects of loss per se. Taken as a whole then, the results of the yoked-control conditions are consistent with the results of Pietras and Hackenberg (2005) and further support the view that response suppression is due in large part to the token-loss contingency rather than to collateral changes in token and food reinforcement.

There were differences worth noting in the effects on response rates of the two yoking procedures. In general, the yoked-food conditions (Part 1) reduced response rates to a greater degree than did the yoked token-loss conditions (Part 2). This may appear counterintuitive, at least from the standpoint of conditioned reinforcement; that is, to the extent that tokens function as conditioned reinforcers, one might expect conditions in which tokens are produced and accumulated (yoked food) to maintain higher response rates than conditions in which tokens are removed nearly as soon as they appear (yoked token loss). The conditioned reinforcing value of the tokens, however, depends on more than their mere presence; it depends on the correlation between the tokens and other reinforcers (exchange stimuli and food). In the yoked-food condition, 


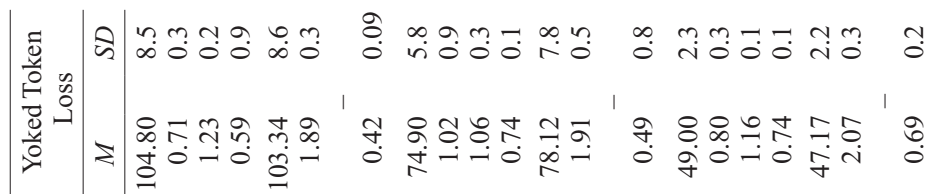

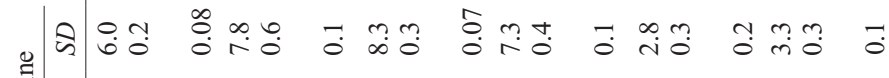

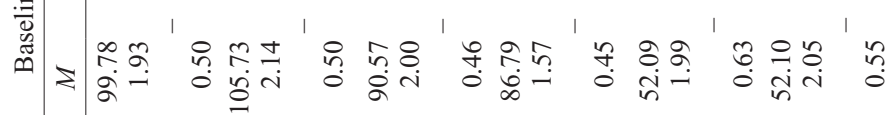

भी

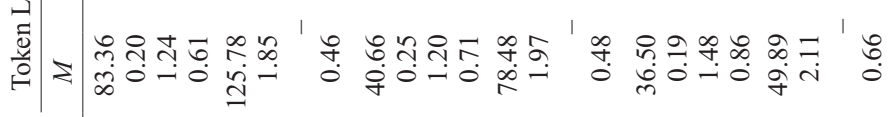

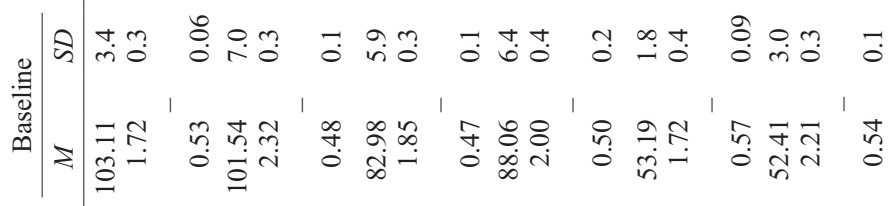

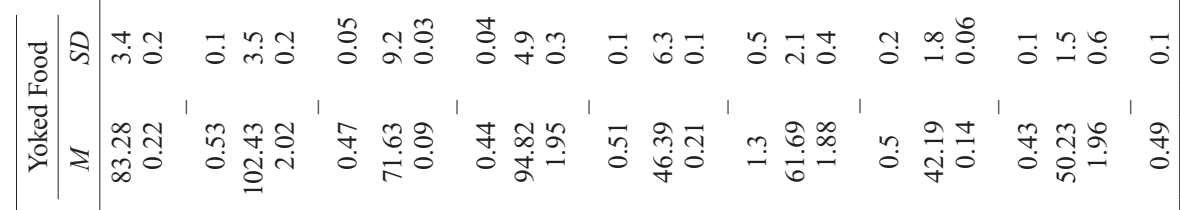

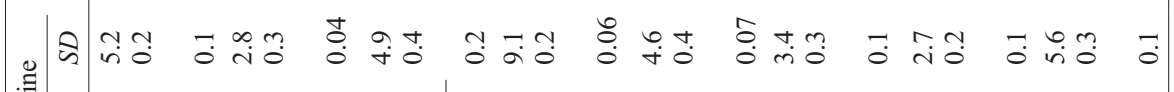

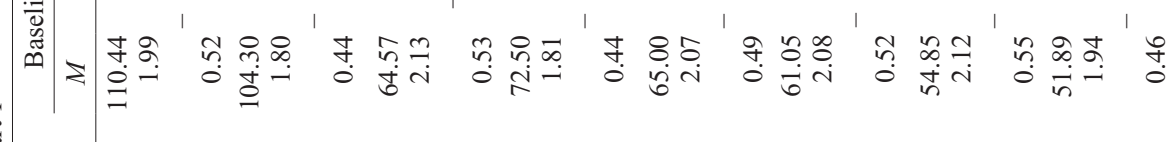

ڤ

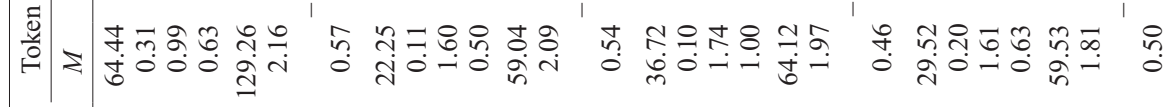

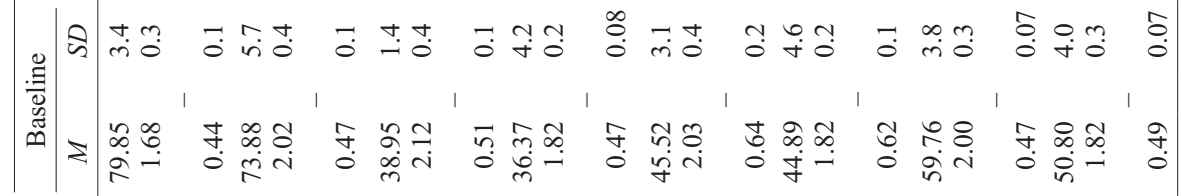

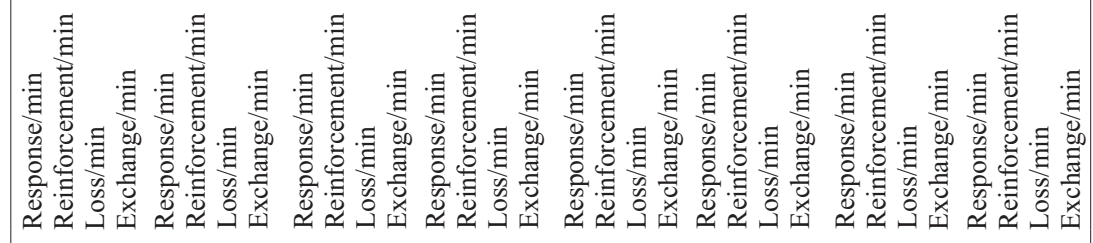

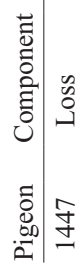

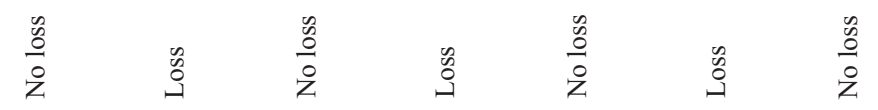



$\stackrel{+}{\stackrel{2}{2}}$

尺े 
an average of four tokens were present during exchange periods but very few were exchangeable for food (a product of the yoking procedure, in which food density was controlled). This may have degraded the reinforcing value of the tokens relative to the yoked token-loss conditions, in which a positive correlation between tokens and food was maintained. It was also true that rates of food reinforcement were somewhat higher under yoked token-loss than under yoked-food conditions, and this variable may have contributed to the differential effects of the two procedures. Comparisons of the two yoking procedures are further complicated by order effects, because the yoked token-loss conditions were always conducted following the yoked-food conditions.

Because the present study was not designed to assess the relative contributions of these variables, such work must await further research. The contribution of the present research lies in demonstrating a clear punishment effect when the rates of food reinforcement (yoked food) and the rates of token loss (yoked token loss) were held constant. A third potential source of reinforcement is that provided by the exchange-period stimuli, stimuli correlated with food across most of the conditions in the experiment. To equate the overall frequency of exchange periods, exchanges were scheduled according to a response-independent schedule during token-loss conditions, but this procedure may have also acted to suppress responding. That is, to the extent that the exchange stimuli functioned as conditioned reinforcers, response-independent presentation of these stimuli may have decreased responding, just as response-independent food has been shown to do (Lattal \& Abreu-Rodrigues, 1997; Sizemore \& Lattal, 1977). This may raise questions about the degree to which decreases in response rate during token-loss conditions were a function of contingent token loss or of response-independent presentations of the exchange stimuli. Although plausible, there are two lines of evidence that argue against this possibility. First, this response-independent exchange schedule was present in both token-loss and yoked token-loss conditions. It seems reasonable to suppose that such a contingency would produce equivalent effects in both conditions, and could not therefore account for the differential suppression in these conditions. Second, as shown in Figure 4, decreases in token-loss conditions were most apparent in the presence of the tokens. No such pattern existed during the yoked token-loss condition, where the same responseindependent exchange schedule was in effect. Furthermore, although not shown here, a similar pattern was evident for Pigeon 774 during the yoked-food condition of Part 1, with approximately equal rates of responding in the presence and absence of tokens (recall that this subject unintentionally experienced response-independent presentations of exchange stimuli during this condition). Together, these data support the conclusion that suppression of response rates during the token-loss conditions were due primarily to the contingent token loss rather than to response-independent exchange stimuli.

In addition to the changes in the target (loss and yoked) components, the contingencies also produced changes in the unaltered (no-loss) components. Such interactions between responding across components are common in multiple schedules (e.g., Catania, 1961; Lander \& Irwin, 1968; Reynolds, 1961), including those involving punishment (Azrin \& Holz, 1966). Prior research has shown, for example, that unpunished responding increases as a function of punishment contingencies arranged in a second component (Brethower \& Reynolds, 1962; Caplan \& Graefe, 1980; Crosbie, Williams, Lattal, Anderson, \& Brown, 1997; Lattal \& Griffin, 1972), a phenomenon termed punishment contrast. Similarly, in the present study, response rates in the no-loss component increased markedly when the token-loss contingency was introduced in the other component. Such changes were less evident in the yoked-food condition of Part 1, despite comparable reinforcement rates, suggesting that the initial contrast may have resulted from the combined effects of the lower reinforcement rates and the token-loss contingency. This interpretation is complicated, however, by order effects, since the contrast effects seen under the initial token-loss conditions did not return when the token-loss conditions were reinstated in Part 2. These results are similar to those of Crosbie et al., who, with pigeons as subjects and electric shock as the punisher, found punishment contrast during the first exposure but not in subsequent exposures to punishment conditions. Crosbie et al. suggested that this transient contrast effect may have been part of a general transition to higher rates of unpunished responding. This shift in no-loss component response rates also occurred in the present experiment (see Table 2).

The cumulative response records (see Figure 5) show that responding was maintained at a fairly constant pattern under both loss and no-loss conditions. The main difference was in the slope of the records: Under token-loss conditions, the slope was shallower than in yoked conditions. That punishment alters mainly the slope rather than the pattern of behavior is consistent with prior research on fixed-ratio punishment, both with token loss (Pietras \& Hackenberg, 2005) and electric shock (Azrin, Holz, \& Hake, 1963) as the punishing stimulus.

One difference between the present findings and those using mild or moderate shock as punishment is the lack of a "warm-up" effect (e.g., Azrin, 1960a, 1960b; Azrin \& Holz, 1966; Hake, Azrin, \& Oxford, 1967), characterized by an initial suppression in response rates early in the session that gradually recovers as the session progresses. This gradual recovery of behavior has also been noted across sessions: Response rates are suppressed to a greater extent during the initial few sessions of shock punishment but gradually recover to approximate baseline levels over the course of a few weeks (Azrin, 1960b; Hake et al., 1967). Neither of these effects was evident in the present results. The slopes of the cumulative records shown in Figure 5 did not change systematically as a function of their temporal location within the session, as would be expected from within-session recovery of behavior. Moreover, across sessions, response suppression was not only rapid (normally occurring within the first 5 sessions of punishment), but stable (remaining relatively suppressed for dozens of daily sessions; see Table 1). 
The present findings also differ from those using highintensity shocks, in which responding is completely suppressed by punishment and remains so even after removal of the punishment contingency (e.g., Appel, 1961; Azrin, 1960a). In the present study, responding was suppressed but not entirely eliminated by the punishment contingency. Moreover, responding quickly recovered to baseline levels when the punishment contingency was removed. Both of these outcomes parallel those reported by Pietras and Hackenberg (2005) with response-cost contingencies. It is unclear at present whether these differences - between response-cost and electric-shock punishment - are qualitative or quantitative - that is, whether more stringent token-loss contingences could be developed to rival the suppression seen with high-intensity shock punishers. Future research might usefully be directed toward this end.

It is generally assumed, but rarely demonstrated, that reinforcement and punishment are symmetrical but opposite in their effects on behavior. The main difficulties are methodological: Qualitative differences in the nature of the reinforcers (food) and punishers (shock) have precluded direct comparisons. Two general approaches have emerged to address this challenge. The first is to scale the events functionally by defining one in relation to the other; for example, by converting shock to negative food units (Farley \& Fantino, 1978). The main limitation of this approach is that it is not possible to specify a priori the rules for scaling positive and negative food units. These parameters must be established empirically and are necessarily post hoc. The second strategy is to restrict the analysis to human subjects only, taking advantage of a preexperimental history with points and/or money and with instructions that establish symmetries between losses and gains (e.g., Critchfield, Paletz, Macaleese, \& Newland, 2003). The main limitations of this approach are that it depends on preexisting verbal histories outside the experimental context and may be limited to verbally proficient organisms. The present study suggests an alternative strategy that relies neither on post hoc scaling nor on preexperimental histories. The use of a token-reinforcement system provides a straightforward and analytically tractable experimental context for examining reinforcement and punishment in conceptually analogous terms as symmetrical changes along a common dimension.

In sum, the present results provide additional support for the proposition that contingent token loss functions as an effective punisher under laboratory conditions. The results extend prior findings with response-cost punishment contingencies, clarifying the conditions under which token loss serves an aversive function. Historically, the study of punishment, and indeed that of aversive control more generally, has relied nearly exclusively on the use of electric shock as an aversive stimulus. There are numerous practical reasons for this, including the calibration and standardization of various dimensions of the stimulus (e.g., intensity, duration, etc.) that are more difficult to accomplish with less commonly used aversive stimuli such as noises, lights, and extreme temperatures (Baron, 1991). At the same time, the study of aversive control needs to ex- pand beyond its traditional reliance on electric shock, encompassing a broader range of aversive stimuli including response-cost and other types of contingencies, in which the aversive functions arise from losses in reinforcement.

\section{AUTHOR NOTE}

This research was supported by NSF Grants SES 998242 and IBN 0420747. Portions of these data have been presented at annual conferences for the Southeastern Association for Behavior Analysis in October 2004 and the Association for Behavior Analysis in May 2004 and May 2007. We thank Anthony DeFulio and Rachelle Yankelevitz for their assistance with this research, and Jesse Dallery for providing feedback on an earlier draft of the manuscript. Address correspondence to B. R. Raiff, Department of Psychology, University of Florida, Gainesville, FL 32611-2250 (e-mail: raiffbr@ufl.edu).

Note-This article was accepted by the previous editorial team, when Shepard Siegel was Editor.

\section{REFERENCES}

APPEL, J. B. (1961). Punishment in the squirrel monkey Saimiri sciurea. Science, 133, 36.

AzRIN, N. H. (1960a). Effects of punishment intensity during variableinterval reinforcement. Journal of the Experimental Analysis of Behavior, 3, 123-142.

Azrin, N. H. (1960b). Sequential effects of punishment. Science, 131, 605-606.

Azrin, N. H., \& Holz, W. C. (1966). Punishment. In W. K. Honig (Ed.), Operant behavior: Areas of research and applications (pp. 380-447). East Norwalk, CT: Appleton-Century-Crofts.

Azrin, N. H., Holz, W. C., \& HaKe, D. F. (1963). Fixed-ratio punishment. Journal of the Experimental Analysis of Behavior, 6, 141-148.

BARON, A. (1991). Avoidance and punishment. In I. H. Iverson \& K. A. Lattal (Eds.), Techniques in the behavioral and neural sciences: Experimental analysis of behavior. Part 1 (pp. 173-217). New York: Elsevier.

Brethower, D. M., \& Reynolds, G. S. (1962). A facilitative effect of punishment on unpunished behavior. Journal of the Experimental Analysis of Behavior, 5, 191-199.

Bullock, C. E., \& HACKEnBerg, T. D. (2006). Second-order schedules of token reinforcement with pigeons: Implications for unit price. Journal of the Experimental Analysis of Behavior, 85, 95-106.

Caplan, M., \& Graefe, J. (1980). Punishment-induced suppression and the occurrence of behavioral contrast in rats with septal lesions. Behavioral \& Neural Biology, 30, 278-291.

CAtania, A. C. (1961). Behavior contrast in multiple and concurrent schedules of reinforcement. Journal of the Experimental Analysis of Behavior, 4, 335-342.

Critchfield, T. S., Paletz, E. M., Macaleese, K. R., \& NewLAND, M. C. (2003). Punishment in human choice: Direct or competitive suppression? Journal of the Experimental Analysis of Behavior, 80, 1-27.

Crosbie, J., Williams, A. M., Lattal, K. A., Anderson, M. M., \& BRown, S. M. (1997). Schedule interactions involving punishment with pigeons and humans. Journal of the Experimental Analysis of Behavior, 68, 161-175.

Farley, J., \& Fantino, E. (1978). The symmetrical law of effect and the matching relation in choice behavior. Journal of the Experimental Analysis of Behavior, 29, 37-60.

Foster, T. A., \& Hackenberg, T. D. (2004). Unit price and choice in a token-reinforcement context. Journal of the Experimental Analysis of Behavior, 81, 5-25.

Foster, T. A., HackenberG, T. D., \& VAidya, M. (2001). Second-order schedules of token reinforcement with pigeons: Effects of fixed- and variable-ratio exchange schedules. Journal of the Experimental Analysis of Behavior, 76, 159-178.

HaCkenberg, T. D., \& Vaidya, M. (2003). Determinants of pigeons' choices in token based self-control procedures. Journal of the Experimental Analysis of Behavior, 79, 207-218. 
HaKe, D. F., AzRin, N. H., \& OXFord, R. (1967). The effects of punishment intensity on squirrel monkeys. Journal of the Experimental Analysis of Behavior, 10, 95-107.

JACKSON, K., \& HACKenberG, T. D. (1996). Token reinforcement, choice, and self-control in pigeons. Journal of the Experimental Analysis of Behavior, 66, 29-49.

KELLEHER, R. T. (1958). Fixed-ratio schedules of conditioned reinforcement with chimpanzees. Journal of the Experimental Analysis of Behavior, 1, 281-289.

KELLEHER, R. T. (1966). Conditioned reinforcement in second-order schedules. Journal of the Experimental Analysis of Behavior, 9, 475-485.

LANDER, D. G., \& IRWIN, R. J. (1968). Multiple schedules: Effects of the distribution of reinforcements between components on the distribution of responses between components. Journal of the Experimental Analysis of Behavior, 11, 517-524.

Lattal, K. A., \& Abreu-Rodrigues, J. (1997). Response-independent events in the behavior stream. Journal of the Experimental Analysis of Behavior, 68, 375-398.

Lattal, K. A., \& Griffin, M. A. (1972). Punishment contrast during free-operant avoidance. Journal of the Experimental Analysis of Behavior, 18, 509-516.

Malagodi, E. F., Webbe, F. M., \& Waddel, T. R. (1975). Second-order schedules of token reinforcement: Effects of varying the schedule of food presentation. Journal of the Experimental Analysis of Behavior, 24, 173-181.
Munson, K. J., \& CROSBIE, J. (1998). Effects of response cost in computerized programmed instruction. Psychological Record, 48, 233-250.

O’Donnell, J., Crosbie, J., Williams, D. C., \& Saunders, K. J. (2000). Stimulus control and generalization of point-loss punishment with humans. Journal of the Experimental Analysis of Behavior, 73, 261-274.

Pietras, C. J., \& Hackenberg, T. D. (2005). Response-cost punishment via token loss with pigeons. Behavioural Processes, 69, 343-356.

REYNOLDS, G. E. (1961). Behavioral contrast. Journal of the Experimental Analysis of Behavior, 4, 57-71.

Sizemore, O. J., \& Lattal, K. A. (1977). Dependency, temporal contiguity, and response-independent reinforcement. Journal of the Experimental Analysis of Behavior, 25, 119-125.

WEINER, H. (1962). Some effects of response cost upon human operant behavior. Journal of the Experimental Analysis of Behavior, 5, 201-208.

WeINER, H. (1963). Response cost and the aversive control of human behavior. Journal of the Experimental Analysis of Behavior, 6 , 415-421.

WEINER, H. (1964). Response cost and fixed-ratio performance. Journal of the Experimental Analysis of Behavior, 7, 79-81.

(Manuscript received August 3, 2007; revision accepted for publication August 24, 2007.) 